\title{
INVARIANT AND PARTIALLY INVARIANT SOLUTIONS WITH RESPECT TO GALILEAN SHIFTS AND DILATATION
}

\author{
E.V. MAKAREVICH
}

\begin{abstract}
In the work we consider a three-dimensional subalgebra embedded in a fourdimensional subalgebra in order to find the set of solutions and to adjoint them with the solutions on subalgebras of higher dimension. Although the aim is not reached yet, we obtain invariant solutions of the rank 1 and partially invariant solutions of the rank 1 and defect 1 . We obtain two submodels being invariant and partially invariant, seven solutions depend on arbitrary function and nineteen exact solutions.
\end{abstract}

Keywords: gas dynamics, hierarchy of submodels, invariant solution, partially invariant solution.

Mathematics Subject Classification: 35Q35, 35B06.

\section{INTRODUCTION}

The equations of gas dynamics (EGD)

$$
\rho D \vec{u}+\nabla p=0, D \rho+\rho \nabla \cdot \vec{u}=0, D S=0,
$$

where $D=\partial_{t}+\vec{u} \cdot \nabla$ is the total derivative w.r.t. the time, $\vec{u}$ is the speed vector, $p$ is the pressure, $\rho$ is the density, $c^{2}=\frac{\partial p}{\partial \rho}$ is the square of the sonic speed and the state equation with the separated density

$$
\rho=h(p) S, \quad S \text { is the entropy function, }
$$

admit twelve-dimensional Lie algebra of operators $L_{12}$ [1]. The optimal system of dissimilar sublalgebras for EGD with state equation (2) is given in [2, Table 3]. By the example of a five-dimensional self-normalized subalgebra, the hierarchy of EGD submodels was considered and the graph of all embedded subalgebras was composed. The submodels were embedded one into another in such a way that the solution of invariant submodel for overalgebra is a partial one to an invariant submodel of overlagebra [3]. In work [4], we constructed and studied a partially invariant solution on a four-dimensional subalgebra from graph $\Gamma_{5}[3]$.

In the present paper we consider a three-dimensional subalgebra embedded into the fourdimensional subalgebra from work [4]. Our aim is to find the set of solutions to adjoin them with solutions on subalgebras of higher dimensions. Although this aim is not attained yet, we however obtain an invariant submodel of rank 1 and all possible partially invariant solutions of rank 1 and defect 1 . We obtain 23 solutions depending on several constants denoted by the letters $v_{0}, w_{0}, \rho_{0}, K_{0}, P_{0}, h_{0}, x_{0}, n, C, C_{i}, i=0,1,2$. All the solutions are written up to the transformations admitted by EGD.

E.V. Makarevich, Invariant and Partially invariant SOlutions With RespeCt to Galilean SHIFTS AND DILATATION.

(C) Makarevich E.V. 2013.

The work is supported by the grant no. 11.G34.31.0042 of Government of Russia under the law no. 220.

Submitted March 6, 2013. 
There is a general fact on the existence of the hierarchy of embedded submodels being invariant, partially-invariant, or differentially-invariant [5].

\section{INVARIANT SOLUTIONS}

Consider a three-dimensional subalgebra 3.23 [2, Table 3] with the basis in the cartesian coordinate system: $\left\{t \partial_{y}+\partial_{v}, t \partial_{z}+\partial_{w}, b\left(t \partial_{t}+x \partial_{x}+y \partial_{y}+z \partial_{z}\right)+t \partial_{t}-u \partial_{u}-v \partial_{v}-w \partial_{w}+2 \rho \partial_{\rho}\right\}$, $b \neq 0, b \neq-1$. The point invariants are calculated in work [3, Table 1]. The solution is represented as

$$
\begin{aligned}
& u=t^{-1}\left(|t|^{\frac{b}{b+1}} u_{1}\left(x_{1}\right)\right), v=t^{-1}\left(|t|^{\frac{b}{b+1}} v_{1}\left(x_{1}\right)+y\right), w=t^{-1}\left(|t|^{\frac{b}{b+1}} w_{1}\left(x_{1}\right)+z\right), \\
& \rho=|t|^{\frac{2}{b+1}} \rho_{1}\left(x_{1}\right), S=|t|^{\frac{2}{b+1}} S_{1}\left(x_{1}\right), p=p\left(x_{1}\right), x_{1}=|t|^{-\frac{b}{b+1}} x .
\end{aligned}
$$

Hereinafter we assume $\rho_{1} \neq 0$, otherwise the solution has no physical meaning. State equation (2) becomes

$$
\rho_{1}=h(p) S_{1}
$$

We introduce the notation

$$
\bar{u}_{1}=u_{1}-b(b+1)^{-1} x_{1},
$$

then substituting (3), (5) into EGD (1) gives the invariant submodel

$$
\begin{aligned}
& p_{x_{1}}=\rho_{1}\left((1-b)(1+b)^{-1} \bar{u}_{1}-\bar{u}_{1} \bar{u}_{1 x_{1}}+b(b+1)^{-2} x_{1}\right), \\
& \bar{u}_{1} v_{1 x_{1}}=-b(b+1)^{-1} v_{1}, \\
& \bar{u}_{1} w_{1 x_{1}}=-b(b+1)^{-1} w_{1}, \\
& \bar{u}_{1} \rho_{1 x_{1}} \rho_{1}{ }^{-1}+\bar{u}_{1 x_{1}}=-(3 b+4)(b+1)^{-1}, \\
& \bar{u}_{1} S_{1 x_{1}}=-2(b+1)^{-1} S_{1} .
\end{aligned}
$$

In studying the submodel, several cases appear. If $\bar{u}_{1}=0$, then $\rho_{1}=0$ and the solution has physical meaning. Let $\bar{u}_{1} \neq 0$. We introduce a new variable $s$ (up to an additive constant) by the formula

$$
d s=\bar{u}_{1}^{-1} d x_{1} .
$$

Integrating system (4), (6), (7), we obtain the set of integrals depending on five constants $v_{0}$, $w_{0}, \rho_{0}, S_{0}, h_{0}$,

$$
\begin{aligned}
& v_{1}=v_{0} e^{-\frac{b}{b+1} s}, w_{1}=w_{0} e^{-\frac{b}{b+1} s}, \rho_{1} x_{1 s}=\rho_{0} e^{-\frac{3 b+4}{b+1} s}, \\
& S_{1}=S_{0} e^{-\frac{2}{b+1} s}, h(p) x_{1 s}=h_{0} e^{-\frac{3 b+2}{b+1} s} .
\end{aligned}
$$

If in the latter identity $h(p) \neq$ const, then the system is reduced to a second order ordinary nonlinear differential equation

$$
\frac{3 b+2}{b+1}+\frac{x_{1 s s}}{x_{1 s}}=\rho_{0} e^{-\frac{3 b+4}{b+1} s}\left(\frac{b-1}{b+1} x_{1 s}+x_{1 s s}-\frac{b}{(b+1)^{2}} x_{1}\right) \varphi\left(\frac{h_{0}}{x_{1 s}} e^{-\frac{3 b+2}{b+1} s}\right),
$$

where we introduce the superposition of functions $\varphi=\left(h^{\prime} h^{-1}\right) \circ h^{(-1)}$, and $\rho_{0}$ is an essential constant which can be made equal to 1 by a dilatation in $x_{1}$.

If $h(p)=$ const, then $x_{1 s}=x_{0} e^{-\frac{3 b+2}{b+1} s}$ and three cases are possible. 
1) $b \neq-\frac{2}{3}, b \neq-\frac{4}{3}$. Then by means of a shift in $s$ we make $x_{0}=1$. The solution to system (6) is determined by the formulae

$$
\begin{aligned}
& u_{1}=-2 x_{1}+\frac{3 b+2}{b+1} C, v_{1}=v_{0}\left|x_{1}-C\right|^{\frac{b}{3 b+2}}, w_{1}=w_{0}\left|x_{1}-C\right|^{\frac{b}{3 b+2}}, \\
& \rho_{1}=\rho_{0}\left|x_{1}-C\right|^{\frac{2}{3 b+2}}, p=\frac{3 b+2}{b+1} \rho_{0}\left|x_{1}-C\right|^{\frac{3 b+4}{3 b+2}}\left(\frac{(b+2)(3 b+2)}{(b+1)(3 b+4)} C-x_{1}\right) .
\end{aligned}
$$

2) $b=-\frac{4}{3}$. By means of a shift in $s$ we obtain $x_{0}=1$. The solution is determined by the formulae

$$
\begin{aligned}
& u_{1}=-2 x_{1}+6 C, v_{1}=v_{0}\left(x_{1}-C\right)^{\frac{2}{3}}, w_{1}=w_{0}\left(x_{1}-C\right)^{\frac{2}{3}}, \\
& \rho_{1}=\rho_{0}\left(x_{1}-C\right)^{-1}, p=-6 \rho_{0} x_{1}-12 \rho_{0} C \ln \left|x_{1}-C\right| .
\end{aligned}
$$

3) $b=-\frac{2}{3}$. Then $x_{1 s}=C, h=h_{0} C^{-1}$. The solution is given by the formulae

$$
\begin{aligned}
& u_{1}=-2 x_{1}+C, v_{1}=v_{0} e^{\frac{2}{C} x_{1}}, w_{1}=w_{0} e^{\frac{2}{C} x_{1}}, \\
& \rho_{1}=\rho_{0} e^{-\frac{6}{C} x_{1}}, p=\rho_{0} C e^{-\frac{6}{C} x_{1}}\left(x_{1}-\frac{2}{3} C\right) .
\end{aligned}
$$

Solutions to EGD in terms of physical variables are provided by formulae (3), in which we substitute (8) with some solution to equation of submodel (9) or (10), (11), (12). In what follows we write only the formulae for $u_{1}, v_{1}, w_{1}, \rho_{1}, p$.

\section{REgUlAR PARTIALLY INVARIANT SOLUtions OF RANK 1 AND DEFECT 1 DEPENDiNG ON ALL SPATIAL VARIABLES}

On subalgebra 3.23 we construct a partially invariant solution of rank 1 and defect 1 . By the expressions for the invariants we find the speed and density, at that, the pressure is assumed to be a general function. The representation for the solution reads as

$$
\begin{aligned}
& u=t^{-1}\left(|t|^{\frac{b}{b+1}} u_{1}\left(x_{1}\right)\right), v=t^{-1}\left(|t|^{\frac{b}{b+1}} v_{1}\left(x_{1}\right)+y\right), w=t^{-1}\left(|t|^{\frac{b}{b+1}} w_{1}\left(x_{1}\right)+z\right), \\
& \rho=|t|^{\frac{2}{b+1}} \rho_{1}\left(x_{1}\right), p=p(x, y, z, t), x_{1}=|t|^{-\frac{b}{b+1}} x .
\end{aligned}
$$

State equation $(2)$ determines the entropy

$$
|t|^{\frac{2}{b+1}} \rho_{1}\left(x_{1}\right)=h(p) S .
$$

Substituting representations (13) and (14) into EGD (1), we determine all the derivatives for the pressure that yields

$$
p=|t|^{-\frac{b}{b+1}}\left(C_{1} y+C_{2} z\right)+P\left(x_{1}\right)+K(t) .
$$

After that, EGD casts into the form of an equations system with one independent variable $x_{1}$

$$
\begin{gathered}
\rho_{1}\left((b+1)^{-1} u_{1}+\left(b(b+1)^{-1} x_{1}-u_{1}\right) u_{1 x_{1}}\right)=P_{x_{1}}, \\
\rho_{1}\left(-b(b+1)^{-1} v_{1}+\left(b(b+1)^{-1} x_{1}-u_{1}\right) v_{1 x_{1}}\right)=C_{1}, \\
\rho_{1}\left(-b(b+1)^{-1} w_{1}+\left(b(b+1)^{-1} x_{1}-u_{1}\right) w_{1 x_{1}}\right)=C_{2}, \\
\rho_{1 x_{1}} \rho_{1}^{-1}\left(b(b+1)^{-1} x_{1}-u_{1}\right)-2(b+1)^{-1}=u_{1 x_{1}}+2,
\end{gathered}
$$

and the functionally overdetermined identity

$$
\begin{array}{r}
h^{\prime} h^{-1}\left((b+1)^{-1}|t|^{-\frac{b}{b+1}}\left(C_{1} y+C_{2} z\right)-\left(b(b+1)^{-1} x_{1}-u_{1}\right) P_{x_{1}}+t K_{t}+\right. \\
\left.+C_{1} v_{1}+C_{2} w_{1}\right)=2(b+1)^{-1}-\rho_{1_{x_{1}}} \rho_{1}^{-1}\left(b(b+1)^{-1} x_{1}-u_{1}\right) .
\end{array}
$$


where $P$ is an arbitrary function of $x_{1}, K$ is an arbitrary function of $t$. Consider the case when the solution depends on all spatial variables: $C_{1}{ }^{2}+C_{2}{ }^{2} \neq 0$. Then by means of identity (20) we exclude the expression

$$
|t|^{-\frac{b}{b+1}}\left(C_{1} y+C_{2} z\right)=p-P\left(x_{1}\right)-K(t)
$$

valid due to 15$)$. We obtain the identity w.r.t. the independent variables $t, x_{1}, p$. We differentiate w.r.t. $t$ to obtain the identity

$$
h^{\prime}\left(-(b+1)^{-1} K+t K_{t}\right)_{t}=0 .
$$

Suppose the first multiplier in (21) is non-zero, $h^{\prime} \neq 0$. In this case, by (21), we determine the function $K=K_{0}|t|^{\frac{1}{b+1}}-C_{0}(b+1), K_{0}, C_{0}$ are constants. The pressure is written as $p=|t|^{-\frac{b}{b+1}}\left(C_{1} y+C_{2} z\right)+P\left(x_{1}\right)+K_{0}|t|^{\frac{1}{b+1}}-C_{0}(b+1)$. Equation 20 becomes

$$
\begin{array}{r}
(b+1)^{-1}(p-P)+C_{0}-\left(b(b+1)^{-1} x_{1}-u_{1}\right) P_{x_{1}}+C_{1} v_{1}+C_{2} w_{1}= \\
=h h^{\prime-1}\left(2(b+1)^{-1}-\rho_{1_{x_{1}}} \rho_{1}^{-1}\left(b(b+1)^{-1} x_{1}-u_{1}\right)\right) .
\end{array}
$$

Here $2(b+1)^{-1}-\rho_{1 x_{1}} \rho_{1}^{-1}\left(b(b+1)^{-1} x_{1}-u_{1}\right) \neq 0$ since otherwise we get a contradiction in identity 22 . We differentiate 22 w.r.t. $p$,

$$
\left(h h^{-1}\right)^{\prime}=(b+1)^{-1}\left(2(b+1)^{-1}-\rho_{1_{x_{1}}} \rho_{1}^{-1}\left(b(b+1)^{-1} x_{1}-u_{1}\right)\right)^{-1} .
$$

In this identity, the variables $x_{1}, p$ separate and thus, both sides of the identity are constant. By the latter identity we find $h=h_{0}\left(p+P_{0}\right)^{\frac{1}{\gamma}}, \gamma \neq 0$. If we replace $p+P_{0}$ by $p$ and $h_{0} S$ by $S$, then $h=p^{\frac{1}{\gamma}}$, the state equation becoms $\rho=p^{\frac{1}{\gamma}} S$. In EGD $(1)$, instead of entropy equation, we can write the equation for the pressure, $D p+\gamma p \nabla \cdot \vec{u}=0$. We substitute function $h$ into (22) and collect the coefficients at the powers of $p$. It leads us to overdetermined equations system (16), (17), 18), as well as

$$
\begin{gathered}
\left(b(b+1)^{-1} x_{1}-u_{1}\right) P_{x_{1}}+(b+1)^{-1} P=C_{1} v_{1}+C_{2} w_{1}+C_{0} \\
\rho_{1_{x_{1}} \rho_{1}} \rho^{-1}\left(b(b+1)^{-1} x_{1}-u_{1}\right)=\left(2-\gamma^{-1}\right)(b+1)^{-1} \\
u_{1 x_{1}}=-\left(2 b+2+\gamma^{-1}\right)(b+1)^{-1}
\end{gathered}
$$

By 25. we find $u_{1}=-\left(2 b+2+\gamma^{-1}\right)(b+1)^{-1} x_{1}+C(b+1)^{-1}, C$ is a constant. Then equation (24) can be rewritten as

$$
\rho_{1_{x_{1}}} \rho_{1}^{-1}\left(\left(3 b+2+\gamma^{-1}\right) x_{1}-C\right)=2-\gamma^{-1} .
$$

Suppose now that $3 b+2+\gamma^{-1}=0$ in 26 . If $\gamma^{-1}=2$, then $b=-\frac{4}{3}$ and 26 reduces to $\rho_{1_{x_{1}}} C=0$. The case $C \neq 0$ leads one to a non-physical solution $\rho=0$. As $C=0$, the solution is determined by the formulae

$$
\begin{aligned}
& u_{1}=4 x_{1}, v_{1}=-\frac{1}{4} C_{1} \rho_{1}{ }^{-1}, w_{1}=-\frac{1}{4} C_{2} \rho_{1}{ }^{-1}, \rho_{1}{ }^{2}=\frac{C_{1}{ }^{2}+C_{2}{ }^{2}}{144\left(-x_{1}{ }^{2}+x_{0}{ }^{2}\right)}, \\
& p=t^{-4}\left(C_{1} y+C_{2} z\right)+\frac{1}{12}\left(C_{1}{ }^{2}+C_{2}{ }^{2}\right) \rho_{1}{ }^{-1}+K_{0}|t|^{-3}, x_{1}=t^{-4} x .
\end{aligned}
$$

If $\gamma^{-1} \neq 2$, by integrating we arrive at the contradiction $C_{1}=C_{2}=0$.

Let $3 b+2+\gamma^{-1} \neq 0$. As $\gamma^{-1}=2$, we obtain four solutions with different values of parameter $b$. 
1) $b=-2$. The solution is determined by the formulae

$$
\begin{aligned}
& u_{1}=-2 C, v_{1}=v_{0}\left(x_{1}+C\right)-\frac{1}{2} C_{1} \rho_{0}{ }^{-1}, w_{1}=w_{0}\left(x_{1}+C\right)-\frac{1}{2} C_{2} \rho_{0}{ }^{-1}, \\
& \rho_{1}=\rho_{0}, p=t^{-2}\left(C_{1} y+C_{2} z\right)+2 \rho_{0} C\left(x_{1}+C\right)+K_{0} t^{-1}+\frac{1}{2} \rho_{0}{ }^{-1}\left(C_{1}{ }^{2}+C_{2}{ }^{2}\right),
\end{aligned}
$$

where $x_{1}=t^{-2} x, C_{1} v_{0}+C_{2} w_{0}=2 \rho_{0} C$.

2) $b=-\frac{8}{5}$. The solution is given by the formulae

$$
\begin{aligned}
& u_{1}=\frac{4}{3} x_{1}, v_{1}=v_{0} x_{1}{ }^{2}-\frac{3}{8} C_{1} \rho_{0}{ }^{-1}, w_{1}=w_{0} x_{1}{ }^{2}-\frac{3}{8} C_{2} \rho_{0}{ }^{-1}, \\
& \rho_{1}=\rho_{0}, p=|t|^{-\frac{8}{3}}\left(C_{1} y+C_{2} z\right)-\frac{2}{9} \rho_{0} x_{1}{ }^{2}+K_{0}|t|^{-\frac{5}{3}}+\frac{9}{40} \rho_{0}{ }^{-1}\left(C_{1}{ }^{2}+C_{2}{ }^{2}\right) .
\end{aligned}
$$

where $x_{1}=|t|^{-\frac{8}{3}} x, C_{1} v_{0}+C_{2} w_{0}+\frac{2}{9} \rho_{0}=0$.

3) $b=-\frac{3}{2}$. The solution is determined by the formulae

$$
\begin{aligned}
& u_{1}=2 x_{1}, v_{1}=v_{0} x_{1}^{3}-\frac{1}{3} C_{1} \rho_{0}^{-1}, w_{1}=w_{0} x_{1}^{3}-\frac{1}{3} C_{2} \rho_{0}{ }^{-1}, \\
& \rho_{1}=\rho_{0}, p=|t|^{-3}\left(C_{1} y+C_{2} z\right)-\rho_{0} x_{1}{ }^{2}+K_{0}|t|^{-2}+\frac{1}{6} \rho_{0}{ }^{-1}\left(C_{1}{ }^{2}+C_{2}{ }^{2}\right) .
\end{aligned}
$$

where $x_{1}=|t|^{-3} x, C_{1} v_{0}+C_{2} w_{0}=0$.

4) $b=-\frac{5}{3}$. The solution is given by the formulae

$$
\begin{aligned}
& u_{1}=x_{1}-\frac{3}{2} C, v_{1}=v_{0}\left(x_{1}+C\right)^{\frac{5}{3}}-\frac{2}{5} C_{1} \rho_{0}^{-1}, w_{1}=w_{0}\left(x_{1}+C\right)^{\frac{5}{3}}-\frac{2}{5} C_{2} \rho_{0}^{-1}, \\
& \rho_{1}=\rho_{0}, p=|t|^{-\frac{5}{2}}\left(C_{1} y+C_{2} z\right)+\frac{15}{4} \rho_{0} C\left(x_{1}+C\right)+K_{0}|t|^{-\frac{3}{2}}+\frac{4}{15} \rho_{0}^{-1}\left(C_{1}^{2}+C_{2}^{2}\right) .
\end{aligned}
$$

where $x_{1}=|t|^{-\frac{5}{2}} x, C_{1} v_{0}+C_{2} w_{0}=0$.

As $\gamma^{-1} \neq 2$, the system is solvable only for the following values of parameters $b$ and $\gamma^{-1}$ :

5) $b=-\frac{3}{2}, \gamma^{-1}=\frac{3}{2}$. The solution is determined by the formulae

$$
\begin{aligned}
& u_{1}=x_{1}-2 C, v_{1}=v_{0}\left|x_{1}+C\right|^{\frac{3}{2}}-\frac{1}{2} C_{1} \rho_{0}^{-1}\left|x_{1}+C\right|^{\frac{1}{2}}, \\
& w_{1}=w_{0}\left|x_{1}+C\right|^{\frac{3}{2}}-\frac{1}{2} C_{2} \rho_{0}^{-1}\left|x_{1}+C\right|^{\frac{1}{2}}, \rho_{1}=\rho_{0}\left|x_{1}+C\right|^{-\frac{1}{2}}, \\
& p=|t|^{-3}\left(C_{1} y+C_{2} z\right)+12 \rho_{0} C\left|x_{1}+C\right|^{\frac{1}{2}}+K_{0}|t|^{-2},
\end{aligned}
$$

where $x_{1}=|t|^{-3} x, C_{1} v_{0}+C_{2} w_{0}=0, C_{1}{ }^{2}+C_{2}{ }^{2}=24 \rho_{0}{ }^{2} C$.

6) $b=-\frac{13}{9}, \gamma^{-1}=\frac{5}{3}$. The solution is given by the formulae

$$
\begin{aligned}
& u_{1}=\frac{7}{4} x_{1}-\frac{3}{2} C, v_{1}=v_{0}\left|x_{1}+C\right|^{\frac{13}{6}}-\frac{2}{5} C_{1} \rho_{0}{ }^{-1}\left|x_{1}+C\right|^{\frac{1}{2}}, \\
& w_{1}=w_{0}\left|x_{1}+C\right|^{\frac{13}{6}}-\frac{2}{5} C_{2} \rho_{0}^{-1}\left|x_{1}+C\right|^{\frac{1}{2}}, \rho_{1}=\rho_{0}\left|x_{1}+C\right|^{-\frac{1}{2}}, \\
& p=|t|^{-\frac{13}{4}}\left(C_{1} y+C_{2} z\right)+\frac{1}{8} \rho_{0}\left|x_{1}+C\right|^{\frac{1}{2}}\left(110 C-7 x_{1}\right)+K_{0}|t|^{-\frac{9}{4}},
\end{aligned}
$$

where $x_{1}=|t|^{-\frac{13}{4}} x, C_{1} v_{0}+C_{2} w_{0}=0, C_{1}^{2}+C_{2}^{2}=\frac{1755}{32} \rho_{0}^{2} C$. 
7) $b=-\frac{8}{5}, \gamma^{-1}=\frac{6}{5}$. The solution is determined by the formulae

$$
\begin{aligned}
& u_{1}=-\frac{8}{3} C, v_{1}=v_{0}\left(x_{1}+C\right)-\frac{3}{4} C_{1} \rho_{0}^{-1}\left|x_{1}+C\right|^{\frac{1}{2}}, \\
& w_{1}=w_{0}\left(x_{1}+C\right)-\frac{3}{4} C_{2} \rho_{0}{ }^{-1}\left|x_{1}+C\right|^{\frac{1}{2}}, \rho_{1}=\rho_{0}\left|x_{1}+C\right|^{-\frac{1}{2}}, \\
& p=|t|^{-\frac{8}{3}}\left(C_{1} y+C_{2} z\right)+\frac{80}{9} \rho_{0} C\left|x_{1}+C\right|^{\frac{1}{2}}+K_{0}|t|^{-\frac{5}{3}},
\end{aligned}
$$

where $x_{1}=|t|^{-\frac{8}{3}} x, C_{1} v_{0}+C_{2} w_{0}=0, C_{1}{ }^{2}+C_{2}{ }^{2}=\frac{320}{81} \rho_{0}{ }^{2} C$.

Let $h(p)=h_{0}$ be constant, then (21) is identically satisfied. In this case we obtain system (16), (17), (18), and $u_{1}=-2 x_{1}+C$,

$$
\rho_{1_{x_{1}} \rho_{1}}{ }^{-1}\left((3 b+2)(b+1)^{-1} x_{1}-C\right)=2(b+1)^{-1} .
$$

There appear extra four cases with different values of parameter $b$.

8) If $b=-\frac{2}{3}$, the latter equation implies $C \neq 0$. The solution is given by the formulae

$$
\begin{aligned}
& u_{1}=-2 x_{1}+C, v_{1}=v_{0} e^{\frac{2}{C} x_{1}}-\frac{1}{4} C_{1} \rho_{0}{ }^{-1} e^{\frac{6}{C} x_{1}}, w_{1}=w_{0} e^{\frac{2}{C} x_{1}}-\frac{1}{4} C_{2} \rho_{0}{ }^{-1} e^{\frac{6}{C} x_{1}}, \\
& \rho_{1}=\rho_{0} e^{-\frac{6}{C} x_{1}}, p=t^{2}\left(C_{1} y+C_{2} z\right)+\rho_{0} C e^{-\frac{6}{C} x_{1}}\left(x_{1}-\frac{2}{3} C\right)+K(t) .
\end{aligned}
$$

where $x_{1}=t^{2} x, K(t)$ is an arbitrary function.

9) If $b=-\frac{4}{3}$, we replace $C$ by $6 C$. The solution is determined by the formulae

$$
\begin{aligned}
& u_{1}=-2 x_{1}+6 C, v_{1}=\left|x_{1}-C\right|^{\frac{2}{3}}\left(v_{0}+\frac{1}{2} C_{1} \rho_{0}{ }^{-1}\left|x_{1}-C\right|^{\frac{1}{3}}\right), \\
& w_{1}=\left|x_{1}-C\right|^{\frac{2}{3}}\left(w_{0}+\frac{1}{2} C_{2} \rho_{0}{ }^{-1}\left|x_{1}-C\right|^{\frac{1}{3}}\right), \rho_{1}=\rho_{0}\left(x_{1}-C\right)^{-1}, \\
& p=t^{-4}\left(C_{1} y+C_{2} z\right)-6 \rho_{0} x_{1}-12 \rho_{0} C \ln \left|x_{1}-C\right|+K(t) .
\end{aligned}
$$

where $x_{1}=t^{-4} x$.

10) $b=-2$. Replace $C$ by $4 C$. The solution is given by the formulae

$$
\begin{aligned}
& u_{1}=-2 x_{1}+4 C, v_{1}=\left|x_{1}-C\right|^{\frac{1}{2}}\left(v_{0}+\frac{1}{4} C_{1} \rho_{0}{ }^{-1} \ln \left|x_{1}-C\right|\right), \\
& w_{1}=\left|x_{1}-C\right|^{\frac{1}{2}}\left(w_{0}+\frac{1}{4} C_{2} \rho_{0}{ }^{-1} \ln \left|x_{1}-C\right|\right), \rho_{1}=\rho_{0}\left|x_{1}-C\right|^{-\frac{1}{2}}, \\
& p=t^{-2}\left(C_{1} y+C_{2} z\right)-4 \rho_{0}\left|x_{1}-C\right|^{\frac{1}{2}} x_{1}+K(t),
\end{aligned}
$$

where $x_{1}=t^{-2} x$.

11) $b \neq-\frac{2}{3}, b \neq-\frac{4}{3}, b \neq-2$. Replace $C$ by $\frac{3 b+2}{b+1} C$. The solution is determined by the formulae

$$
\begin{aligned}
& u_{1}=-2 x_{1}+\frac{3 b+2}{b+1} C, v_{1}=v_{0}\left|x_{1}-C\right|^{\frac{b}{3 b+2}}-\frac{b+1}{b+2} C_{1} \rho_{0}{ }^{-1}\left|x_{1}-C\right|^{-\frac{2}{3 b+2}} \\
& w_{1}=w_{0}\left|x_{1}-C\right|^{\frac{b}{3 b+2}}-\frac{b+1}{b+2} C_{2} \rho_{0}^{-1}\left|x_{1}-C\right|^{-\frac{2}{3 b+2}}, \rho_{1}=\rho_{0}\left|x_{1}-C\right|^{\frac{2}{3 b+2}} \\
& p=|t|^{-\frac{b}{b+1}}\left(C_{1} y+C_{2} z\right)+\frac{3 b+2}{b+1} \rho_{0}\left|x_{1}-C\right|^{\frac{3 b+4}{3 b+2}}\left(\frac{(b+2)(3 b+2)}{(b+1)(3 b+4)} C-x_{1}\right)+K(t) .
\end{aligned}
$$




\section{REgUlar PARTIALLY INVARIANT SOLUtions DEPENDING ON ONE SPATIAL VARIABLE}

Let $C_{1}=C_{2}=0, p=P\left(x_{1}\right)+K(t)$. If $K(t)=$ const, we obtain the invariant submodel considered in Section 2 and this is why we let $K(t) \neq$ const. We introduce the notation by formula (5), then EGD yield the differential equations

$$
\begin{gathered}
P_{x_{1}}=\rho_{1}\left((1-b)(b+1)^{-1} \bar{u}_{1}-\bar{u}_{1} \bar{u}_{1 x_{1}}+b(b+1)^{-2} x_{1}\right), \\
\bar{u}_{1} v_{1 x_{1}}=-b(b+1)^{-1} v_{1}, \\
\bar{u}_{1} w_{1 x_{1}}=-b(b+1)^{-1} w_{1}, \\
\bar{u}_{1} \rho_{1_{x_{1}}} \rho_{1}^{-1}+\bar{u}_{1 x_{1}}=-(3 b+4)(b+1)^{-1},
\end{gathered}
$$

and also the overdetermined identity

$$
h^{\prime} h^{-1}\left(\bar{u}_{1} P_{x_{1}}+t K_{t}\right)=\bar{u}_{1} \rho_{1 x_{1}} \rho_{1}^{-1}+2(b+1)^{-1} .
$$

If $P=P_{0}$ is constant, then in (43) the variables $t, x_{1}$ separate and are determined $K(t)$ : $h\left(K(t)+P_{0}\right)=h_{0}|t|^{\frac{C}{b+1}}$. By $\sqrt{42}, \sqrt{390}$ it follows $\bar{u}_{1}=-b(b+1)^{-1} x_{1}$ or $\bar{u}_{1}=(b+1)^{-1} x_{1}$. In the former case, solution to (39)-(43) read as

$$
u_{1}=0, v_{1}=v_{0} x_{1}, w_{1}=w_{0} x_{1}, \rho_{1}=\rho_{0}\left|x_{1}\right|^{\frac{2 b+4}{b}}, p=K(t)+P_{0} .
$$

In the latter we get

$$
u_{1}=x_{1}, v_{1}=v_{0}\left|x_{1}\right|^{-b}, w_{1}=w_{0}\left|x_{1}\right|^{-b}, \rho_{1}=\rho_{0}\left|x_{1}\right|^{-3 b-5}, p=K(t)+P_{0} .
$$

In what follows, we assume $P \neq$ const. We have $\bar{u}_{1} P_{x_{1}}+t K_{t} \neq 0$ in equation (43), overwise we arrive at the contradiction $K(t)=$ const. If at the same time $h(p)=h_{0}$ is constant, then this case coincides with the similar one in Section 3 as $C_{1}=C_{2}=0$. If $h^{\prime} \neq 0$, it follows from 43 that $\bar{u}_{1} \rho_{1_{x_{1}}} \rho_{1}^{-1}+2(b+1)^{-1} \neq 0$. We differentiate 43 w.r.t. $t$ and $x_{1}$,

$$
\begin{gathered}
\left(\frac{h^{\prime}}{h}\right)^{\prime}\left(\bar{u}_{1} P_{x_{1}}+t K_{t}\right)+\frac{h^{\prime}}{h}\left(1+t \frac{K_{t t}}{K_{t}}\right)=0, \\
\left(\frac{h^{\prime}}{h}\right)^{\prime}\left(\bar{u}_{1} P_{x_{1}}+t K_{t}\right) P_{x_{1}}+\frac{h^{\prime}}{h}\left(\bar{u}_{1} P_{x_{1}}\right)_{x_{1}}=\left(\bar{u}_{1} \frac{\rho_{1 x_{1}}}{\rho_{1}}\right)_{x_{1}} .
\end{gathered}
$$

We substitute (43), (46) into (47), divide by $P_{x_{1}}$ and differentiate w.r.t. $x_{1}$,

$$
\left(\frac{\left(\bar{u}_{1} P_{x_{1}}\right)_{x_{1}}}{P_{x_{1}}}\right)_{x_{1}}=\left(\frac{\bar{u}_{1}\left(\bar{u}_{1}\left(\ln \rho_{1}\right)_{x_{1}}\right)_{x_{1}}}{\bar{u}_{1}\left(\ln \rho_{1}\right)_{x_{1}}+2(b+1)^{-1}}\right)_{x_{1}}+\left(\frac{\left(\bar{u}_{1}\left(\ln \rho_{1}\right)_{x_{1}}\right)_{x_{1}}}{P_{x_{1}}\left(\bar{u}_{1}\left(\ln \rho_{1}\right)_{x_{1}}+2(b+1)^{-1}\right)}\right)_{x_{1}} t K_{t} .
$$

The variables in 48 separate.

Suppose the coefficient at $t K_{t}$ is non-zero, then $K(t)=\ln |t|$ and the variables in (43) separate. By change $(7)\left(\right.$ as $\left.\bar{u}_{1} \neq 0\right)$ we obtain integrals and a submodel from one second order nonlinear ordinary differential equation

$$
\begin{gathered}
v_{1}=v_{0} e^{-\frac{b}{b+1} s}, w_{1}=w_{0} e^{-\frac{b}{b+1} s}, \rho_{1} x_{1 s}=\rho_{0} e^{-\frac{3 b+4}{b+1} s} \\
h=h_{0} e^{n p}, p=\ln |t|-n^{-1} \ln \left|x_{1 s}\right|-\left((3 b+2) n^{-1}(b+1)^{-1}+1\right) s, \\
n^{-1}\left(\frac{3 b+2}{b+1}+\frac{x_{1 s s}}{x_{1 s}}\right)+1=\rho_{0} e^{-\frac{3 b+4}{b+1} s}\left(\frac{b-1}{b+1} x_{1 s}+x_{1 s s}-\frac{b}{(b+1)^{2}} x_{1}\right) .
\end{gathered}
$$

If the coefficient at $t K_{t}$ in $(48)$ vanishes, then $\left(\ln \rho_{1}\right)_{s s}=C_{1} P_{s}\left(\left(\ln \rho_{1}\right)_{s}+2(b+1)^{-1}\right)$. System (39)-(43) is solvable if and only if $C_{1}=0$, otherwise by complicated arguments we would arrive at the contradiction. If $\bar{u}_{1} \neq 0$, we introduce variable $s$ by formula (7), and then by (40)-(42) we find $\rho_{1}=\rho_{0} e^{\frac{C-2}{b+1} s}, \bar{u}_{1}=u_{0} e^{-\frac{3 b+2+C}{b+1} s}$, and $v_{1}, w_{1}$ are found by the formulae in 49 . 
If $3 b+2+C \neq 0$, then $x_{1}=-(b+1)(3 b+2+C)^{-1} e^{-\frac{3 b+2+C}{b+1} s}+x_{0}$ and thus

$$
\begin{aligned}
& \bar{u}_{1}=-(3 b+2+C)(b+1)^{-1}\left(x_{1}-x_{0}\right), v_{1}=v_{0}\left|x_{1}-x_{0}\right|^{\frac{b}{3 b+2+C}}, \\
& w_{1}=w_{0}\left|x_{1}-x_{0}\right|^{\frac{b}{3 b+2+C}}, \rho_{1}=\rho_{0}\left|x_{1}-x_{0}\right|^{-\frac{C-2}{3 b+2+C}}
\end{aligned}
$$

with new constants $v_{0}, w_{0}, \rho_{0}$. Due to 460,47$)$ we get the identity $\frac{\left(\bar{u}_{1} P_{x_{1}}\right)_{x_{1}}}{P_{x_{1}}}=1+t \frac{K_{t t}}{K_{t}}=C_{0}$, $C_{0}$ is a constant. If $C_{0} \neq 0$, then $p=C_{1}\left|x_{1}-x_{0}\right|^{-\frac{C_{0}(b+1)}{3 b+2+C}}+C_{2}|t|^{C_{0}}+P_{0}$, and by (43) we find $h(p)=h_{0}\left|p-P_{0}\right|^{\frac{C}{C_{0}(b+1)}}$. We substitute the found solution into 39 and study the compatibility to obtain three solutions/.

1) $C_{0}=-\frac{6 b+6+C}{b+1}$. The solution is determined by the formulae

$$
\begin{aligned}
& u_{1}=-(2 b+2+C)(b+1)^{-1} x_{1}, v_{1}=v_{0}\left|x_{1}\right|^{\frac{b}{3 b+2+C}}, w_{1}=w_{0}\left|x_{1}\right|^{\frac{b}{3 b+2+C}}, \\
& \rho_{1}=\rho_{0}\left|x_{1}\right|^{-\frac{C-2}{3 b+2+C}}, p=C_{1}\left|x_{1}\right|^{\frac{6 b+6+C}{3 b+2+C}}+C_{2}|t|^{-\frac{6 b+6+C}{b+1}}+P_{0},
\end{aligned}
$$

where $C_{1}$ is given by the identity $C_{1}(6 b+6+C)(b+1)^{2}=\rho_{0}(2 b+2+C)(3 b+3+C)(3 b+2+C)$.

2) $C=-2(b+1)$. The solution is determined by the formulae

$$
\begin{aligned}
& u_{1}=b(b+1)^{-1} x_{0}, v_{1}=v_{0}\left(x_{1}-x_{0}\right), w_{1}=w_{0}\left(x_{1}-x_{0}\right), \\
& \rho_{1}=\rho_{0}\left|x_{1}-x_{0}\right|^{\frac{2 b+4}{b}}, p=C_{1}\left|x_{1}-x_{0}\right|^{-\frac{C_{0}(b+1)}{b}}+C_{2}|t|^{C_{0}}+P_{0},
\end{aligned}
$$

where $C_{1}$ is given by the identity $C_{1} C_{0}(b+1)^{3}=b \rho_{0} x_{0}(3 b+2+C)$.

3) $C=-3(b+1)$. The solution is determined by the formulae

$$
\begin{aligned}
& u_{1}=x_{1}-(b+1)^{-1} x_{0}, v_{1}=v_{0}\left|x_{1}-x_{0}\right|^{-b}, w_{1}=w_{0}\left|x_{1}-x_{0}\right|^{-b}, \\
& \rho_{1}=\rho_{0}\left|x_{1}-x_{0}\right|^{-3 b-5}, p=C_{1}\left|x_{1}-x_{0}\right|^{C_{0}(b+1)}+C_{2}|t|^{C_{0}}+P_{0},
\end{aligned}
$$

where $C_{1}$ is given by the identity $C_{1} C_{0}(b+1)^{3}=b \rho_{0} x_{0}(3 b+2+C)$.

If $C_{0}=0$, then $p=-C_{1}(b+1)(3 b+2+C)^{-1} \ln \left|x_{1}-x_{0}\right|+C_{2} \ln |t|+P_{0}$. By (43) we find $h(p)=h_{0} e^{\frac{C}{(b+1)\left(C_{1}+C_{2}\right)}}$. We substitute the found functions into 39 and consider the solvability to get extra three solutions.

4) $C=-6(b+1)$. The solution is determined by the formulae

$$
\begin{aligned}
& u_{1}=4 x_{1}-\frac{3 b+4}{b+1} x_{0}, v_{1}=v_{0}\left|x_{1}-x_{0}\right|^{-\frac{b}{3 b+4}}, w_{1}=w_{0}\left|x_{1}-x_{0}\right|^{-\frac{b}{3 b+4}}, \\
& \rho_{1}=\rho_{0}\left|x_{1}-x_{0}\right|^{-2}, p=\frac{C_{1}(b+1)}{3 b+4} \ln \left|x_{1}-x_{0}\right|+C_{2} \ln |t|+P_{0},
\end{aligned}
$$

where $C_{1}(b+1)=12 \rho_{0}(3 b+4)$.

5) $b=-\frac{4}{3}, C=-\frac{4}{3}$. The solution is determined by the formulae

$$
\begin{aligned}
& u_{1}=4 x_{0}, v_{1}=v_{0}\left(x_{1}-x_{0}\right), w_{1}=w_{0}\left(x_{1}-x_{0}\right), \rho_{1}=\rho_{0}\left(x_{1}-x_{0}\right)^{-1}, \\
& p=-\frac{1}{4} C_{1} \ln \left|x_{1}-x_{0}\right|+C_{2} \ln |t|+P_{0},
\end{aligned}
$$

6) $b=-\frac{4}{3}, C=-1$. The solution is determined by the formulae

$$
\begin{aligned}
& u_{1}=x_{1}+3 x_{0}, v_{1}=v_{0}\left|x_{1}-x_{0}\right|^{\frac{4}{3}}, w_{1}=w_{0}\left|x_{1}-x_{0}\right|^{\frac{4}{3}}, \rho_{1}=\rho_{0}\left|x_{1}-x_{0}\right|^{-1}, \\
& p=-\frac{1}{3} C_{1} \ln \left|x_{1}-x_{0}\right|+C_{2} \ln |t|+P_{0} .
\end{aligned}
$$


If $3 b+2+C=0$, then $x_{1}=$ const, the contradiction.

If $\bar{u}_{1}=0$, then $b=-\frac{4}{3}$. We obtain the solution

$$
u_{1}=4 x_{1}, v_{1}=0, w_{1}=0, p=\ln |t|+P\left(x_{1}\right), \rho_{1}=-\frac{1}{12} x_{1}^{-1} P_{x_{1}},
$$

where $P_{x_{1}}$ is an arbitrary function.

\section{Conclusion}

On subalgebra 3.23, by the optimal system in work [2], we obtain two submodels: (9) is an invariant one of rank 1 and (50) is partially invariant submodel of rank 1 and defect 1 . We also obtain seven solutions (35), (36), (37), (38), (44), (45), (58), depending on one arbitrary functions and exact solutions (10)-(12), (27)-(34), (44), (45), (52)-(57) depending on several constants. The minimal amount of the constants is four, the maximal one is eight.

\section{BIBLIOGRAPHY}

1. L.V. Ovsyannikov. The "podmodeli" program. Gad dynamics // Prikl. matem. mekhan. 1992. V. 58, No. 4. P. 30-55. [J. Appl. Math. Mech. 1992. V. 58, No. 4. P. 601-627.]

2. E.V. Makarevich. Optimal system of subalgebras admitted by the gas dynamics equations in the case of state equation with separated density // Siber. Elect. Math. Rep. 2011. V. 8. P. 19-38. (in Russian).

3. E.V. Makarevich. Gasdynamics equations submodels hierarchu in case of state equation with separated density // Siber. Elect. Math. Rep. 2012. V. 9. P. 306-328. (in Russian).

4. E.V. Makrevich. Collapse or instant source of gas on straight line // Ufimskij matem. zhur. 2012. V. 4, No. 4. P. 119-129. [Ufa Math. J. 2012. V. 4, No. 4. P.116-126.]

5. S.V. Khabirov. Hierarchy of submodels of differential equations // Archives of ALGA. 2012. V. 9. P. 79-94.

Elena Vladimirovna Makarevich,

Ufa State Aviation Technical University,

K. Marx str., 12

450000, Ufa, Russia

E-mail: Makarevich_EV@mail.ru 